\title{
Complexity of large time behaviour of evolution equations with bounded data
}

\author{
Juan Luis Vázquez \\ Departamento de Matemáticas \\ Universidad Autónoma \\ 28049 Madrid. Spain. \\ and \\ Enrique Zuazua \\ Departamento de Matemática Aplicada \\ Universidad Complutense \\ 28040 Madrid. Spain
}

May 8, 2001

\begin{abstract}
We study the asymptotic behaviour of solutions of the heat equation and a number of evolution equations using scaling techniques. We prove that in the framework of bounded data stabilization need not occur and the general asymptotic behaviour is complex. This behaviour reflects for large times, even on compact sets, the complexity of the initial data at infinity.
\end{abstract}

AMS Subject Classification: 35B40, 35K60, 35K65.

Keywords and phrases: asymptotic behaviour, scaling, omega limit, heat equation, hyperbolic conservation laws. 


\section{Introduction}

In recent decades much attention has been paid to the study of the long time behaviour of suitable classes of solutions of partial differential equations using the concepts of dynamical systems. Such concepts, like orbit, omega limit and attractor, were introduced in the first half of the century for the investigation of systems of ordinary differential equations, which are finite dimensional instances of dynamical systems, and were successfully extended to the infinite dimensional framework.

Here we want to combine these ideas with the property of invariance under the scaling group to obtain results on the complex large time behaviour of a number of evolution equations with a simple structure when they are posed in the set of bounded measurable functions defined in the whole space, $L^{\infty}\left(\mathbb{R}^{N}\right)$. Invariance under the scaling group allows the equation to copy in certain way the behaviour of the initial data at infinity of the space variable into the behaviour of the solution $u=u(\cdot, t)$ for large $t$. The results apply to the classical heat equation, which we take as a model, but also to a large number of evolution equations like the porous medium equation, the $p$-Laplacian equation, the wave equation, and scalar conservation laws.

Scale invariance is at the heart of our argument. However, it is really necessary only in a weak form called quasi-invariance. This will allow our theory to be extended to homogenization problems, equations involving reaction, convection and diffusion, and the equations of fluid mechanics.

The space $L^{\infty}\left(\mathbb{R}^{N}\right)$ plays an important role in providing for a setting where complexity occurs in the asymptotic behaviour of the above-mentioned equations. Thus, the evolution problems under consideration are usually well-posed in other functional spaces like $L^{p}\left(\mathbb{R}^{N}\right)$, for some $1<p<\infty$, and the asymptotic behaviour can be then rather simple, reflecting the simple structure of the equation. Consider for instance the heat equation. When we consider solutions defined in $\mathbb{R}^{N}$ it is well known that every solution with initial data $u_{0} \in L^{1}\left(\mathbb{R}^{N}\right)$ converges as $t \rightarrow \infty$ towards a multiple of the fundamental solution, the one which has the same integral,

$$
u(x, t) \rightarrow C t^{-N / 2} \exp \left(-\frac{x^{2}}{4 t}\right), \quad C=(4 \pi)^{-N / 2} \int u_{0}(x) d x .
$$

When the space domain is a bounded set $\Omega \subset \mathbb{R}^{N}$ and we take homogeneous Dirichlet conditions the typical space is $L^{2}(\Omega)$, the convergence to a unique type of profile takes place after multiplying by $e^{\lambda_{1} t}$, where $\lambda_{1}$ is the so-called first eigenvalue. A similar result happens for homogeneous Neumann conditions. In the setting of almost periodic functions solutions posed in $\mathbb{R}^{N}$ converge to the space average of the initial data. Similar simplicity occurs for many of the equations mentioned above. We will prove below that, on the contrary, the simple behaviour is lost when $L^{\infty}\left(\mathbb{R}^{N}\right)$ is taken as the functional setting.

The rest of this paper is organized as follows. In Section 2 we present the main results and techniques developed in this paper in the simple example of the linear heat equation. 
Section 3 contains the analysis of the behavior of the initial data under scaling. In Section 4 we present the extension to an abstract setting of the results of Section 2 on the heat equation. In Section 5 we return to the heat equation to make some earlier results more precise. Section 6 is devoted to nonlinear heat equations as the porous media equation and the $p$-Laplacian one. In Section 7 we analyze the interesting case of scalar hyperbolic conservation laws in one and several space dimensions. In Section 8 we extend the analysis done in $L^{\infty}$ to other $L^{p}$ spaces to cover other existing results. In Section 9 we point out that the compactness requirements may be often avoided in the context of linear evolution equations. Finally, in section 10, we observe that most of the results of this paper can be adapted to situations were the equation under consideration is not invariant under the scaling transformation but is quasi-invariant in an appropriate sense that we define.

\section{The heat equation}

In order to introduce the methods and results in a simple setting we consider in the first place the linear constant-coefficient heat equation posed in $\mathbb{R}^{N}$ with $N \geq 1$

$$
\begin{cases}u_{t}=\Delta u & \text { in } \quad Q=\mathbb{R}^{N} \times(0, \infty), \\ u(x, 0)=u_{0}(x) & \text { in } \quad \mathbb{R}^{N}\end{cases}
$$

We want to analyze the asymptotic behaviour of solutions as $t \rightarrow \infty$. It is easy to see that for suitable initial data there exists a constant $c \in \mathbb{R}$ such that

$$
u(\cdot, t) \rightarrow c \quad \text { in } \quad L^{\infty}\left(\mathbb{R}^{N}\right)
$$

as $t \rightarrow \infty$. This happens at least in two cases.

(i) When $u_{0}=c+f$ with $c \in \mathbb{R}$ and $f \in L^{p}\left(\mathbb{R}^{N}\right)$ for some $p \in[1, \infty)$, and

(ii) when $u_{0}$ is periodic.

In the first case the solution converges to $c$ with a polynomial rate, in the second one the asymptotic $c$ is the average of the initial datum on a periodicity cell and the convergence rate is exponential. Obviously, the constant is not the only possible asymptotic regime. This can be easily seen considering for instance

(iii) initial data in $1 \mathrm{D}$ with two different asymptotic values $c_{ \pm}$at $\pm \infty$. In this case it can be proved that the asymptotic behaviour is given by the special solution of the heat equation with piece-wise constant initial data of Heaviside type, $u_{0}(x)=H(x)$ with

$$
H(x)=c_{+} \quad \text { for } \quad x>0 \quad\left(\text { resp. } H(x)=c_{-} \text {for } \quad x<0\right),
$$

cf. end of this section.

The first goal of this paper is to describe what are all the possible asymptotic regimes of the solutions of the heat equation and other evolution equations for bounded initial data. 
It is natural to address this problem by means of scaling techniques. Given a solution $u(x, t)$ of the heat equation and a constant $\lambda>0$ we introduce the rescaled function

$$
u_{\lambda}(x, t)=u\left(\lambda x, \lambda^{2} t\right) .
$$

The key point is that $u_{\lambda}$ solves the heat equation with initial data

$$
u_{0, \lambda}(x)=u_{0}(\lambda x)
$$

Due to the Maximum Principle we have for every $(x, t)$

$$
|u(x, t)| \leq\left\|u_{0}\right\|_{L^{\infty}\left(\mathbb{R}^{N}\right)},
$$

and the same bound applies to $u_{\lambda}$ for all $\lambda>0$, i.e., it is uniform in $\lambda$. By standard compactness results for the solutions of the heat equation we conclude that along a subsequence $\lambda_{n} \rightarrow \infty$ we have

$$
\begin{aligned}
& u_{0, \lambda} \rightarrow \phi \quad \text { in } \quad L^{\infty}\left(\mathbb{R}^{N}\right) \text { weak-star } \\
& u_{\lambda} \rightarrow v \quad \text { in } \quad L_{l o c}^{\infty}(Q) .
\end{aligned}
$$

Moreover, the limit function $v$ solves the heat equation with initial data $\phi$. In particular, for $t=1$ we have

$$
u_{\lambda}(x, 1) \rightarrow v(x, 1) \quad \text { in } \quad L^{\infty}(K)
$$

uniformly on any compact subset $K \subset \mathbb{R}^{N}$. This can be re-written as follows

$$
u\left(\lambda x, \lambda^{2}\right) \rightarrow v(x, 1) \quad \text { in } \quad L^{\infty}(K) .
$$

By setting $\lambda^{2}=t$ we deduce the convergence of the rescaled orbit

$$
u(\sqrt{t} x, t) \rightarrow v(x, 1) \quad \text { in } \quad L^{\infty}(K)
$$

as $t \rightarrow \infty$ along a suitable subsequence.

Let us now denote by $S_{t}$ the semigroup generated by the heat equation, defined by $\left(S_{t} u_{0}\right)(x)=u(x, t)$. Then,

$$
v(x, 1)=S_{1} \phi
$$

Summing up, we obtain the following result

Theorem 1 The set of accumulation points in $L_{\text {loc }}^{\infty}\left(\mathbb{R}^{N}\right)$ of $u(\sqrt{t} x, t)$ as $t \rightarrow \infty$ coincides with the set $\left\{S_{1}(\phi)\right\}$, where $\phi$ ranges over the set of accumulation points as $\lambda \rightarrow \infty$ of the family $\left\{u_{0, \lambda}: \lambda>0\right\}$ in the weak-star topology of $L^{\infty}\left(\mathbb{R}^{N}\right)$.

Proof. The direct part has already been settled. For the converse, suppose that $u$ is a bounded solution $u(x, t)$ defined for $x \in \mathbb{R}^{N}$ and $t>0$, and assume that we have a sequence $t_{n} \rightarrow \infty$ such that

$$
u\left(\sqrt{t_{n}} x, t_{n}\right) \rightarrow v(x) \quad \text { in } \quad L_{l o c}^{\infty}\left(\mathbb{R}^{N}\right) .
$$


We can write this as

$$
u_{\lambda_{n}}(x, 1) \rightarrow v(x) \quad \text { in } \quad L_{l o c}^{\infty}\left(\mathbb{R}^{N}\right)
$$

with $t_{n}=\lambda_{n}^{2}$. On the other hand, by standard theory, a bounded solution $u$ has a bounded initial trace $u_{0}$ at $t=0$. But the family of rescalings of the initial data $u_{0, \lambda_{n}}(x)=u_{0}\left(\lambda_{n} x\right)$ is bounded in $L^{\infty}\left(\mathbb{R}^{N}\right)$, hence relatively compact in the weak-star topology. This means that, along a finer subsequence,

$$
u_{0, \lambda_{n}}(x) \rightarrow \phi(x)
$$

for some $\phi \in L^{\infty}\left(\mathbb{R}^{N}\right)$. By the direct proof we know that $v=S_{1}(\phi)$.

Remarks. The time $t=1$ in $S_{1}$ is taken for convenience and can be replaced by $S_{\tau}$ for any $\tau>0$ after changing the function $u(\sqrt{t} x, t)$ into $u\left((t / \tau)^{1 / 2} x, t\right)$. In any case, it must be pointed out that the map $S_{1}$ has a regularizing effect on the set of accumulation points of $u_{0, \lambda}$ : all functions of the form $S_{1}(\phi)$ are $C^{\infty}$ smooth and enjoy certain a priori bounds, cf. Section 5.

Therefore, the key point in our project is understanding the set of accumulation points (i.e., accumulation functions) of the family $u_{0, \lambda}$. The following section will be devoted to analyze this question. We will show that in the setting of bounded data the situation can be quite complex. We advance a remarkable result, which will be proved in that section.

Theorem 2 Given any bounded sequence $\left\{g_{j}: j=1,2, \cdots\right\}$ in $L^{\infty}\left(\mathbb{R}^{N}\right)$ there exists a bounded function $u_{0} \in L^{\infty}\left(\mathbb{R}^{N}\right)$ such that the set of accumulation points of the family $\left\{u_{0, \lambda}\right\}_{\lambda}$ as $\lambda \rightarrow \infty$ in the weak-star topology of $L^{\infty}\left(\mathbb{R}^{N}\right)$ contains the whole sequence $\left\{g_{j}\right\}$.

As we will see, the set of accumulation points that contains an infinite sequence will be larger than just this set of points, indeed it can contain a continuum. As an immediate consequence of the two previous results we have

Corollary 3 For every bounded sequence $\left\{\phi_{j}: j=1,2, \cdots\right\}$ in $L^{\infty}\left(\mathbb{R}^{N}\right)$ there exists a bounded function $u_{0} \in L^{\infty}\left(\mathbb{R}^{N}\right)$ such that, if $u(x, t)$ is the corresponding solution of the heat equation, the set of accumulation points of $u(\sqrt{t} x, t)$ as $t \rightarrow \infty$ in $L_{\text {loc }}^{\infty}\left(\mathbb{R}^{N}\right)$ contains the family $\left\{S_{1}\left(\phi_{j}\right)\right\}$.

To conclude this section we note that the particular results on the asymptotic behaviour mentioned at the beginning of the section can be easily understood in this framework. In the case (i) when $u_{0}=c+f$ with $f \in L^{1}\left(\mathbb{R}^{N}\right) \cap L^{\infty}\left(\mathbb{R}^{N}\right)$ the unique limit of $u_{0, \lambda}$ is the constant $c$, and the corresponding solution of the rescaled problems in the limit is $v=c$. On the other hand, when $u_{0}$ is periodic, the unique accumulation point of $u_{0, \lambda}$ is the average of the initial data on the periodicity cell and the corresponding solution $v$ is this constant. Finally, in example (iii) the asymptotic limit has the form $v(x, t)=V\left(x t^{-1 / 2}\right)$ where $V=S_{1} H, H$ being the Heaviside function (2.2). 


\section{The scaling of bounded data}

Let us now return to general theory. We are interested in describing the possible behaviour of bounded functions at infinity using as a tool the action of the scaling group $G=$ $\left\{G_{\lambda}\right\}_{\lambda>0}$. $G$ acts on the space $X=L^{\infty}\left(\mathbb{R}^{N}\right)$ by the formula a

$$
G_{\lambda}(f)(x)=f(\lambda x)
$$

Definition 1. We define the scaling omega-limit set of any function $f$, or $G$-omega-limit set, as the set $G_{\infty}(f)$ of all accumulation points of the family $G_{\lambda}(f)$ as $\lambda \rightarrow \infty$ in the weak-star topology of $L^{\infty}\left(\mathbb{R}^{N}\right), \sigma\left(L^{\infty}, L^{1}\right)$. In the usual notation of dynamical systems we write

$$
G_{\infty}(f)=\bigcap_{r>1} \overline{\gamma_{r}^{+}(f)}
$$

where $\gamma_{r}^{+}(f)$ is the forward $G$-orbit of $f$ starting at $\lambda=r, \gamma_{r}^{+}(f)=\left\{G_{\lambda}(f): \lambda \geq r\right\}$ and the closure is taken in the weak-star topology.

Since the space $L^{1}\left(\mathbb{R}^{N}\right)$ is separable, this topology restricted to a ball $\mathcal{B}_{c}=\{f \in$ $\left.L^{\infty}\left(\mathbb{R}^{N}\right):|g| \leq c\right\}$ is metrizable and we can characterize the $G_{\infty}$-limit sequentially. Thus, $G_{\infty}(f)$ is the set of functions $g \in L^{\infty}\left(\mathbb{R}^{N}\right)$ such that there exists a sequence $\lambda_{n} \rightarrow \infty$ and $G_{\lambda_{n}} f \rightarrow g$ weakly-star. We also have

Lemma 4 The group acts continuously on $X$ endowed with the weak star convergence. It follows easily that the $G$-omega-limit of a function $f \in X$ is never empty. It is also closed and connected in the weak-star topology. It is also invariant under the action of the group.

Proof. The first assertion means that the map $G: X \times \mathbb{R}_{+} \rightarrow X$ is continuous when $X$ is endowed with the weak-star topology. Since weak-star topologies are not so frequent in the literature on asymptotic phenomena, we are giving a proof for the reader's convenience. Firstly, if we restrict $G$ to be defined on a bounded subset of $X$ (as is the case in the application of the present paper) the topology is metrizable and we only need to check that for a bounded sequence $f_{n}$ converging weakly-star to $f$, a sequence of numbers $\lambda_{n}$ converging to $\lambda>0$, and a test function $\phi \in L^{1}\left(\mathbb{R}^{N}\right)$ we have $\int G_{\lambda_{n}}\left(f_{n}\right) \phi d x \rightarrow$ $\int G_{\lambda}(f) \phi d x$. We write the difference as

$$
\int G_{\lambda_{n}}\left(f_{n}\right) \phi d x-\int G_{\lambda}(f) \phi d x=\int f_{n}\left(G_{1 / \lambda_{n}} \phi-\phi\right) d x+\int\left(f_{n}-f\right) \phi d x .
$$

The first term tends to zero because $\phi$ is integrable and the $f_{n}$ are uniformly bounded, while the second converges because $f_{n} \rightarrow f$.

When $G$ is defined on the whole space $X$ we have to replace the sequence $f_{n}$ by a net $f_{\alpha}$ converging to $f$ weakly-star. But then the Banach-Steinhaus theorem implies that the 
family $f_{\alpha}$ is uniformly bounded and we are back in the previous situation. In particular, the decomposition (3.3) with $f_{\alpha}$ instead $f_{n}$ allows to prove just in the same way that $\int G_{\lambda_{n}}\left(f_{\alpha}\right) \phi d x \rightarrow \int G_{\lambda}(f) \phi d x$ as $f_{\alpha} \rightarrow f$ and $\lambda_{n} \rightarrow \lambda$.

The consequences are standard in the dynamical systems literature, cf. [21, 22]. The last assertion means that for every $\lambda>0$ we have $G_{\lambda}\left(G_{\infty}(f)\right)=G_{\infty}(f)$.

Remark. Though we will use by default the topology of weak-star convergence in $L^{\infty}\left(\mathbb{R}^{N}\right)$, we will use the term bounded subset of $L^{\infty}\left(\mathbb{R}^{N}\right)$ in the usual uniform sense unless mention to the contrary.

3.II Simple $G$-omega-limits. We say that the omega-limit is simple if it contains only one function. Particular examples of simple omega-limits have been mentioned in the previous section. For case (i) it is clear that all functions $f \in L^{p}\left(\mathbb{R}^{N}\right) \cap L^{\infty}\left(\mathbb{R}^{N}\right)$, $1 \leq p<\infty$, have a simple omega-limit, $G_{\infty}(f)=\{0\}$. In case (ii) all periodic functions have a constant as simple omega-limit. This is also true for the more general class of almost periodic functions $\mathcal{A}$ characterized as the closure in $L^{\infty}\left(\mathbb{R}^{N}\right)$ (with uniform converge) of set of trigonometric polynomial with arbitrary frequencies,

$$
P(x)=\sum_{1}^{n} a_{k} e^{i \omega_{k} \cdot x}
$$

with $\omega_{k} \in \mathbb{R}^{N}$, [4]. For a function $f \in \mathcal{A}$ the $G_{\infty}$-set is given by the limit of the averages

$$
G_{\infty}(f)=\lim _{R \rightarrow \infty} \oint_{B_{R}} f d x
$$

On the other hand, a non-constant omega-limit is given by the piecewise-constant initial data in one dimension.

A consequence of the invariance of the omega-limit is the following characterization of simple omega-limits.

Lemma 5 If an omega-limit is simple, $G_{\infty}(f)=\{g\}$, then $g$ has the form $g(x)=$ $g(x /|x|)$.

It is immediate that this $g$ is its own $\omega$-limit, $g=G_{\infty}(g)$, though any bounded function $f$ which coincides with $g$ for large $x$ could be used to obtain $G_{\infty}(f)=g$.

Simple omega-limits for the initial data of an evolution process of the type considered in this paper give rise to evolutions which stabilize to a unique asymptotic profile. We are interested in addressing quite the opposite situation.

3.III. General $G$-omega-limits. At the next level of complexity we construct an example that we call log-periodic. It is based on any function $f \in L^{\infty}\left(\mathbb{R}^{N}\right)$ such that for some real number $a>1$

$$
f(a x)=f(x), \quad x \in \mathbb{R}^{N} .
$$


In that case the $G$-omega limit is given by the log-periodic orbit

$$
G_{\infty}(f)=\left\{G_{\lambda}(f): 1 \leq \lambda \leq a\right\}
$$

that contains $f$ and its scalings, and it is topologically equivalent to a circle, $S^{1}$. An easy way of constructing such functions is to consider spherical coordinates $x=(r, \phi)$, take a bounded function $f_{1}(\phi)$ defined on the sphere $S^{N-1}$ and put

$$
f(r, \phi)=f_{1}(R(r)(\phi))
$$

where $R(r)$ is a family of transformations of the sphere such that $R(1)=i d$ and $R(a r)=$ $R(r)$ for every $r>0$, i.e., $R$ is log-periodic.

As we announced in Theorem 2, we can construct very large omega-limits. We recast the result in the present notation

Lemma 6 Given any sequence $\left\{g_{j}\right\}$ of functions which is bounded in $L^{\infty}\left(\mathbb{R}^{N}\right)$ we can construct a function $f \in L^{\infty}\left(\mathbb{R}^{N}\right)$ such that $G_{\infty}(f)$ contains the closure of that sequence in the weak-star topology, $G_{\infty}(f) \supset \operatorname{clo}\left\{g_{1}, g_{2}, \cdots\right\}$.

Proof. We use a zooming method. Given the function $g_{i}, i \geq 1$, we restrict it to the annulus $A_{j}=\left\{2^{-j}<|x|<2^{j}\right\}, j \geq 1$ integer, and transfer this image to a far away distance by means of a scaling with factor $1 / \lambda_{i j}$

$$
g_{i j}(x)=g_{i}\left(x / \lambda_{i j}\right),
$$

which is defined in the annulus $A_{i j}=\left\{2^{-j} \lambda_{i j}<|x|<2^{j} \lambda_{i j}\right\}$. Next, we select the factors $\lambda_{i j}$ in such a way that all these sets are disjoint. This can be obtained as follows: we first arrange the indexes $(i, j)$ in a sequence by standard diagonal process, and then choose the sequence $\lambda_{i j}$ iteratively so that each annulus lies immediately outside of the preceding one. We can also leave gaps between successive annuli if we wish, only this makes the $\lambda_{i j}$ even larger. We then define the desired $f$ on each annulus $A_{i j}$ by

$$
f(x)=g_{i}\left(x / \lambda_{i j}\right), \quad x \in A_{i j} .
$$

This formula gives then a unique value for $f$ on $\bigcup_{i j} A_{i j}$ since the annuli $A_{i j}$ are disjoint. If there exist gaps between successive annuli we define $f$ on the gaps as zero or in any other bounded way.

It is then clear that applying the group action $G_{\lambda}$ to $f$ with the sequence of factors $\lambda_{i j}$, for fixed $i$ and variable $j=1,2, \cdots$, we obtain in the limit exactly the function $g_{i}$ on the annulus $A_{j}$. Since the sequence $A_{j}$ expands as $j \rightarrow \infty$ we get along this sequence $G_{\lambda}(f) \rightarrow g_{i}$ with uniform convergence on compact subsets of $\mathbb{R}^{N} \backslash\{0\}$. This in particular implies weak-star convergence in $\mathbb{R}^{N}$.

3. IV. Maximal omega-limits. As a corollary of this result and the fact that the unit ball in $L^{\infty}\left(\mathbb{R}^{N}\right)$ is separable in the weak-star topology we have the following consequence. 
Corollary 7 Given $C>0$ there exists a function $f \in L^{\infty}\left(\mathbb{R}^{N}\right)$ with $\|f\|_{\infty}=C$ such that

$$
G_{\infty}(f)=\left\{f \in L^{\infty}\left(\mathbb{R}^{N}\right):\|f\|_{\infty} \leq C\right\} .
$$

Let us look a bit closer at this kind of functions. We define the set

$$
\mathcal{M}=\left\{f \in L^{\infty}\left(\mathbb{R}^{N}\right):\|f\|=1, G_{\infty}(f)=B_{1}\left(L^{\infty}\left(\mathbb{R}^{N}\right)\right)\right\} .
$$

where $B_{1}\left(L^{\infty}\left(\mathbb{R}^{N}\right)\right)$ is the unit ball in $L^{\infty}\left(\mathbb{R}^{N}\right)$. Then we have

Theorem 8 The set $\mathcal{M}$ is dense with empty interior in $B_{1}\left(L^{\infty}\left(\mathbb{R}^{N}\right)\right)$ with the weak-star topology.

Proof. A basis of open neighbourhoods of a function $f \in L^{\infty}\left(\mathbb{R}^{N}\right)$ has the form

$$
B\left(\phi_{1}, \cdots, \phi_{n}, \epsilon\right)=\left\{g \in L^{\infty}\left(\mathbb{R}^{N}\right):\left|\int(f-g) \phi_{i} d x\right| \leq \epsilon \quad \forall i\right\},
$$

where $\phi_{i} \in L^{1}\left(\mathbb{R}^{N}\right), i=1, \ldots N$, and $\epsilon>0$. This condition does not depend on the behaviour of $g$ at far away distances as long as we have a bound like $|g| \leq C$. But the behaviour for large $x$ is precisely what determines $G_{\infty}(f)$.

\section{General evolution setting. Main Asymptotic For- mula}

If we consider again the argument used in Section 2 for the classical heat equation, we can see that there are two types of properties of the equation on which the proof is based, namely (I) the heat equation defines a semigroup with some good properties, and (II) this semigroup is invariant under the action of the scaling group $G$.

In order to set up a framework that applies to a number of linear or nonlinear evolution models we introduce the following abstract setting. Instead of concentrating on the evolution equation

$$
u_{t}=A u,
$$

we look rather to the fact that it generates a semigroup. We assume that

(I.i) The equation generates a point-wise continuous semigroup in $X=L^{\infty}\left(\mathbb{R}^{N}\right)$, i.e., given any initial data $u_{0} \in X$ there exists a unique solution $u \in Y=C([0, \infty): X)$ that we write as $S_{t}\left(u_{0}\right)$ to denote the dependence on the initial data. Then

$$
S: X \times[0, \infty) \rightarrow X, \quad S\left(u_{0}, t\right)=S_{t}\left(u_{0}\right)
$$

is the semigroup map. When we want to stress the dependence on $x$ we write $\left(S_{t} u_{0}\right)(x)=$ $u(x, t)$, and sometimes we write $u(t)$ when no confusion arises. $X$ is endowed with the weak-star topology $\sigma\left(L^{\infty}, L^{1}\right)$. We impose still an additional condition 
(I.ii) For every $t>0$ the map $S_{t}$ is continuous in the weak-star topology of $X=L^{\infty}\left(\mathbb{R}^{N}\right)$. Conditions (I.i) and (I.ii) imply that the map $S$ is separately continuous in both variables. (II) We impose another type of condition on the semigroup, namely scale invariance. By this we mean that there is a power function $\sigma(\lambda)=\lambda^{\alpha}, \alpha>0$, such that whenever $u(x, t)$ is a solution in $Y$ with initial data $u_{0}$ then

$$
\tilde{u}=u(\lambda x, \sigma(\lambda) t)
$$

is also a solution in $Y$, with data $\tilde{u}_{0}=G_{\lambda} u_{0}$. In the case of the heat equation $\sigma(\lambda)=\lambda^{2}$. This property is expressed in functional terms as $S_{t}\left(G_{\lambda}\left(u_{0}\right)\right)=G_{\lambda}\left(S_{\sigma(\lambda) t}\left(u_{0}\right)\right)$, or in abridged form as the commutation rule:

$$
S_{t} G_{\lambda}=G_{\lambda} S_{\sigma(\lambda) t} .
$$

The next stage is to note that we are interested in the convergence on expanding sets of the form $\{|x| \leq c \psi(t)\}$. This makes it convenient to define the rescaled orbit corresponding to a standard orbit $u(\cdot, t)=S_{t} u_{0}$ as

$$
U(y, t)=u(\psi(t) y, t),
$$

where $\psi=\sigma^{-1}$ is the inverse function of $\sigma, \psi(t)=t^{1 / \alpha}$. We denote the "renormalized semigroup" as

$$
\left(R_{t} u_{0}\right)(y)=U(y, t),
$$

which implies the identity: $R_{t}=G_{\psi(t)} S_{t}$. Notice that $R_{1}=S_{1}$. The invariance under $G_{\lambda}$ takes a much simpler form in rescaled form. If $\tilde{U}$ is the rescaled orbit corresponding to initial data $\tilde{u}_{0}(x)=u_{0}(\lambda x)$ then, since $\tilde{U}(y, t)=\tilde{u}(\psi(t) y, t)=u(\lambda \psi(t) y, \sigma(\lambda) t)$, we have

$$
\tilde{U}(y, t)=U(y, \sigma(\lambda) t), \quad \text { i.e. }, \quad R_{t} G_{\lambda} u_{0}=R_{\sigma(\lambda) t} u_{0} .
$$

We define the omega limit of the $R$-evolution orbit in a similar way to what we did for the scaling group $G$, but now we use $R_{t}$ instead of $G_{\lambda}$.

Definition 2. The $R$-omega limit set of the evolution orbit $\left\{R_{t}\left(u_{0}\right)=U(\cdot, t) t>0\right\}$ starting at $u_{0} \in X$ is the set

$$
R_{\infty}\left(u_{0}\right)=\left\{g \in X: \exists t_{n} \rightarrow \infty \text { and } U\left(\cdot, t_{n}\right) \rightarrow g\right\},
$$

with convergence in the topology of $X$.

The basic properties are easily established: the $R$-omega limit set is a nonvoid, closed, connected subset of $X$. Our result relates the omega limits of the scaling group $G_{\lambda}$ and the scaled evolution $R_{t}$.

Theorem 9 (Main Asymptotic Formula) If $S_{t}$ is a semigroup fulfilling properties (I) and (II) above, then for every $u_{0} \in X$ we have

$$
R_{\infty}\left(u_{0}\right)=S_{1}\left(G_{\infty}\left(u_{0}\right)\right) .
$$


Proof. It repeats the lines of Theorem 1. Let $g \in G_{\infty}\left(u_{0}\right)$ and let $\lambda_{n} \rightarrow \infty$ be such that $G_{\lambda_{n}}\left(u_{0}\right) \rightarrow g$ weak-star. We note that

$$
R_{\sigma(\lambda)} u_{0}=R_{1}\left(G_{\lambda} u_{0}\right)=S_{1}\left(G_{\lambda} u_{0}\right) .
$$

Using property (I.ii) we conclude that along the sequence $t_{n}=\sigma\left(\lambda_{n}\right)$

$$
\lim _{n \rightarrow \infty} R_{t_{n}} u_{0}=\lim _{n \rightarrow \infty} S_{1}\left(G_{\lambda_{n}} u_{0}\right)=S_{1}(g) .
$$

Thus, $R_{\infty}\left(u_{0}\right) \subset S_{1}\left(G_{\infty}\left(u_{0}\right)\right)$. The converse is similar.

We conclude that the orbit $R_{t}\left(u_{0}\right)$ stabilizes to a limit only if $G_{\infty}\left(u_{0}\right)$ is simple. Using the fact that $G_{\infty}\left(u_{0}\right)$ is closed under the action of $G$ and that the renormalized semigroup $R_{t}$ commutes with $G$ in the sense of (4.6) we have

Corollary 10 Given $u_{0} \in X$ and given $g \in G_{\infty}\left(u_{0}\right)$ then

$$
R_{t}(g) \in R_{\infty}\left(u_{0}\right) \quad \text { for every } t>0 .
$$

Thus, the whole renormalized orbit of $g$ is contained in the omega-limit of $u_{0}$, proving in this way that the asymptotic behaviour of $u(x, t)$ can oscillate strongly even at a fixed point, say, $x=0$. Summing up, the results show that the evolution equation does not stabilize to a certain asymptotic profile for general initial data in $X$, but it rather copies the complexity of the $G$-omega limit which, as we have seen, can be quite wild.

4.II. Regularizing semigroups. In many cases we have the extra condition on the semigroup:

(I.iii) The semigroup is regularizing. By this we mean that $S_{t}$ maps bounded sets of $X$ into relatively compact subsets in a better space $X_{1} \subset X$ for $t>0$. In the case of the heat equation $X_{1}$ is the set $B C\left(\mathbb{R}^{N}\right)$ of continuous and bounded functions with the topology of uniform convergence on compacts. Then the convergence in the definition of $R_{\infty}\left(u_{0}\right)$ is uniform over compact subsets of $\mathbb{R}^{N}$.

4.III. Local and global convergence. We insist that the local convergences that we have established for the orbit of the semigroup are uniform on compact sets of the variables $y=\sqrt{t} x$. In particular, when the semigroup is regularizing we may consider the space variable $x$ ranging over a fixed bounded set $A \subset \mathbb{R}^{N}$, and then we obtain the following uniform convergence result.

Theorem 11 Let $S_{t}$ be a regularizing semigroup under the above hypotheses and let $u(x, t)$ be an orbit with initial data $u_{0} \in L^{\infty}\left(\mathbb{R}^{N}\right)$. Ten for every $g \in G_{\infty}\left(u_{0}\right)$ there exists a sequence $t_{n} \rightarrow \infty$ such that

$$
u\left(x, t_{j}\right) \rightarrow v(0,1) \quad \text { as } \quad t \rightarrow \infty
$$

uniformly on compact sets $A \subset \mathbb{R}^{N}$, where $v$ is the solution of the heat equation with initial data $g$. 
In particular, there exist bounded initial data for which the set of accumulation points of $\{u(0, t)\}$ as $t \rightarrow \infty$ is the closed interval $[a, b] \subset \mathbb{R}$. It suffices to take as set $g_{j}$ in Lemma 6 the set of constant functions $g_{j}(x)=r_{j}$, where $r_{j}$ ranges over a dense set in $[a, b]$. The same applies to the $\omega$-limit of $\{u(x, t)\}$ for fixed $x \in \mathbb{R}^{N}$.

On the hand, the orbits with complex asymptotics cannot have uniform convergence since the behaviour that will appear at later stages of the evolution must be present at any smaller $t$, typically as $|x| \rightarrow \infty$.

4.IV. Log-periodic solutions. Corresponding to log-periodic data, $u_{0}(a x)=u_{0}(x)$ for some $a>0$, we have a solution $u(x, t)$ such that $u(a x, \sigma(a) t)=u(x, t)$. In terms of the rescaled orbits $U(x, t)=u(\sqrt{t} x, t)$ we have

$$
U(x, \sigma(a) t)=U(x, t) .
$$

This means that the rescaled orbit is $\log$-periodic in time with $\log$-period $T=\sigma(a)$. Recall that this is the same as saying that it is periodic in logarithmic time $\tau=\log (t)$. In this case we can control the speed of recurrence of the orbit around the different points of the omega-limit, which is not true for more complex situations.

\section{The heat equation revisited}

Since the heat equation generates a regularizing semigroup, the results of the last sections apply to say that the set of accumulation points of the renormalized orbit $U(x, t)=$ $u(\sqrt{t} x, t)$, which we will denote by $R_{\infty}\left(u_{0}\right)$, is the image by $S_{1}$ of the set of accumulation points $G_{\infty}\left(u_{0}\right)$. The convergence to the omega-limit $R_{\infty}\left(u_{0}\right)$ takes place locally uniformly in $x$ along subsequences $t_{n} \rightarrow \infty$.

Range constraints. We note that $R_{\infty}\left(u_{0}\right)$ can be a very large set. The maximum extension of such a set is the image of a ball in $L^{\infty}\left(\mathbb{R}^{N}\right)$. We have to add that, since the evolution copies the set $G_{\infty}\left(u_{0}\right)$ in a regularized way given by the map $S_{1}$, this reduces the range of $R_{\infty}\left(u_{0}\right)$ since $S_{1}$ has strong regularizing properties. In other words, there are some constraints for a function to belong to $R_{\infty}\left(u_{0}\right)$ for some $u_{0}$. In the first place, the solutions of the heat equation are $C^{\infty}$ functions. On the other hand, there are quantitative aspects: thus, for every $u_{0} \geq 0$ there is an a priori second-order estimate

$$
\Delta \log (u) \geq-\frac{N}{2 t}
$$

see Aronson and Bénilan [1]. This implies a universal constraint on the range of any omega limit set $R_{\infty}\left(u_{0}\right)$.

Corollary 12 For every $g \in R_{\infty}\left(u_{0}\right)$ with $u_{0} \geq 0$ we have

$$
\Delta \log (g) \geq-\frac{N}{2}
$$


There is a version of this result for any bounded set in $L^{\infty}\left(\mathbb{R}^{N}\right)$, not just for positive solutions, since a lower bound of the form $u \geq-c$ can be converted into $v \geq 0$ by defining $v=u+c$ which is again a solution of the heat equation. In this way, we obtain a lower bound for $\Delta \log (g+c)$ instead of $\Delta \log (g)$.

Let us remark that the extent of this regularization depends on the amount of renormalization. Thus, a renormalization with larger compression, using a function of the form

$$
u\left(c y t^{1 / 2}, t\right), \quad c>1,
$$

instead of Definition 4.4, produces a renormalized semigroup whose omega limit satisfies

$$
R_{\infty}^{\prime}\left(u_{0}\right)=S_{1 / c^{2}}\left(G_{\infty}\left(u_{0}\right)\right)
$$

which is just a rescaled version of the previous one, but this time the evolution is shorter for $c>1$ and the regularization smaller. Thus, the a priori bound from below on the Laplacian of $\log (u)$ is now $-c^{2} N / 2$. In the limit $c \rightarrow \infty$ we recover increasingly the whole range of $G_{\infty}$.

\section{Nonlinear heat equations}

The above general setting applies perfectly not only to the heat equation, but also to the most popular models of quasilinear heat equations, namely the porous medium equation and the $p$-Laplacian equation.

6.I The porous medium and related equations. We will restrict here consideration to the subset of nonnegative solutions, hence $X=L_{+}^{\infty}\left(\mathbb{R}^{N}\right)$, which is the case treated in detail in the literature because of its applications, and avoids technical difficulties. It is well-known (cf. [2]) that the equation

$$
u_{t}=\Delta\left(u^{m}\right), \quad m>0,
$$

defines a semigroup of contractions in $L^{1}\left(\mathbb{R}^{N}\right)$ and can be extended to a much wider class of initial data, in particular to $L_{+}^{\infty}\left(\mathbb{R}^{N}\right)$. The equation is called the porous medium equation for $m>1$, the fast diffusion equation for $0<m<1$ and it reduces to the classical heat equation for $m=1$.

Let us examine the properties of the evolution process. In all cases $m>0$, for every $u_{0} \in L^{\infty}\left(\mathbb{R}^{N}\right), u_{0} \geq 0$ there exists a unique continuous weak solution $u(x, t)$, i.e., $u \in B C(Q) \cap C\left([0, \infty): L_{l o c}^{1}\left(\mathbb{R}^{N}\right)\right)$ and

$$
\iint\left\{u \eta_{t}+u^{m} \Delta \eta\right\} d x d t+\int u_{0}(x) \eta(x, 0) d x=0
$$

holds for every test function $\eta \in C^{2,1}(Q), \eta \geq 0$, which is compactly supported in $\mathbb{R}^{N} \times$ $[0, \infty)$. Hence, the equation generates a semigroup in this class of solutions and property 
(I.i) holds. Also, the Maximum Principle holds. The closure property (I.ii) holds easily: the limits of bounded weak solutions are still bounded weak solutions and weak-star converging initial data pass to the limit in formula (6.2). This uses the fact that the map $S_{t}$ is regularizing, property (I.iii), since the images of bounded sets are relatively compact subsets of $C^{\alpha}$ for some $\alpha>0$ in compact sets of $\mathbb{R}^{N},[10]$. Finally, scale invariance holds with $\sigma(\lambda)=\lambda^{2}$. We then have

Theorem 13 The results of Theorems 9 and 11 apply to this problem and the convergence in the definition of $R_{\infty}$, formula (4.7), takes place uniformly on compact sets.

The possible range of the omega limit set $R_{\infty}\left(u_{0}\right)$ is now controlled by the constraints

$$
\Delta\left(u^{m-1}\right) \geq-\frac{C}{t}, \quad u_{t} \geq-\frac{C u}{t},
$$

which hold for $m>1$, while for $(N-2) / N<m<1$ we have even stronger constraints

$$
\left|\Delta\left(u^{m-1}\right)\right| \leq \frac{C}{t}, \quad\left|u_{t}\right| \leq \frac{C u}{t} .
$$

These a priori bounds have universal constants $C=C(m, N)>0$ independent of the solution under consideration (under the only restriction that $u \geq 0$ ), [1], [6]. For instance, when $0<m<1$ we have

$$
\Delta\left(u^{m-1}\right) \geq \frac{m}{t}
$$

Let us finally remark that some of the results can be extended to the very fast diffusion range $m<0$, where the equation is written as

$$
u_{t}=\nabla \cdot\left(u^{m-1} \nabla u\right),
$$

cf. for instance [33] and references. More generally, we can consider the general filtration equation

$$
u_{t}=\Delta \Phi(u),
$$

where $\Phi$ is a continuous increasing real function, or even a maximal monotone graph in $\mathbb{R}^{2}$, cf. [2]. In order not to lengthen the presentation we restrain from entering into the specific details of these extensions. Let us only assert that the general theory of Section 4 applies under suitable conditions on $\Phi$.

6.II The $p$-Laplacian equation. The equation reads

$$
u_{t}=\operatorname{Div}\left(|\nabla u|^{p-2} \nabla u\right), \quad p>1 .
$$

The properties of the evolution are similar, [11], and a regularizing semigroup is obtained. Scale-invariance holds with $\sigma(\lambda)=\lambda^{p}$. The regularizing effect holds: $S_{t}$ takes bounded 
initial data into solutions with are $C^{1+\alpha}$ in $x, C^{\beta}$ in time for $t \geq \tau>0$. Regularizing estimates have been proved in [16] and take the form for $p>2$

$$
\Delta_{p} v \geq-\frac{C}{t}, \quad u_{t} \geq-\frac{C u}{t},
$$

where $v=u^{(p-2) /(p-1)}$ and $C>0$ is a universal constant that depends only on $p$ and $N$. The estimate is valid for all nonnegative solutions $u=u(x, t)$. But the restriction to positive data is here irrelevant since the equation is invariant under vertical displacements $u \rightarrow u+c$

\section{Scalar conservation laws. The compact case}

We consider in this section the asymptotics of scalar conservation laws in one and more space variables. We start with the one-dimensional theory which relies on better known results and requires less assumptions on the non-linearity.

7.I One space dimension. We study the first-order equation

$$
u_{t}+f(u)_{x}=0
$$

where $u(x, t)$ is a scalar function and $f$ is a locally Lipschitz continuous scalar function. We may assume that $f(0)=0$. The proper concept of solution is given by the entropy solutions that can be formulated as those bounded distributional solutions that satisfy a family of entropy inequalities. We take from Kruzhkov [19, 20] the precise definition of solution.

Definition. An entropy solution of equation (7.1) is a function $u \in L_{l o c}^{\infty}(Q)$ satisfying

$$
\frac{\partial}{\partial t}|u-k|+\frac{\partial}{\partial x}\{\operatorname{sign}(u-k)(f(u)-f(k))\} \leq 0
$$

in $\mathcal{D}^{\prime}(Q)$ for any $k \in \mathbb{R}$. An entropy solution of the Cauchy problem with initial data $u(x, 0)=u_{0}(x) \in L^{\infty}(\mathbb{R})$ is an entropy solution such that

$$
u(\cdot, t) \rightarrow u_{0} \quad \text { in } L_{l o c}^{1}(\mathbb{R}) \quad \text { as } t \rightarrow 0 \text { essentially. }
$$

The Kruzhkov condition (7.2) can be equivalently formulated as two conditions: (i) the equation is satisfied in the sense of distributions, (ii) for very entropy pair $(\phi, \psi)$ where $\phi$ is a convex function and $\psi^{\prime}(u)=f^{\prime}(u) \phi^{\prime}(u)$ we have

$$
\frac{\partial}{\partial t} \phi(u)+\frac{\partial}{\partial x} \psi(u) \leq 0
$$

in the sense of distributions, cf. [28]. 
Kruzhkov proves that the bounded entropy solution of the initial problem exists and is unique, and we have the local estimate for any two such solutions

$$
\int_{B_{t}}|u(x, t)-v(x, t)| d x \leq \int_{B_{0}}\left|u_{0}(x)-v_{0}(x)\right| d x,
$$

where $B_{0}=B(0, R)$ is the ball of center 0 and radius $R>0$ and $B_{t}=B(0, R(t))$ is the ball of radius $R(t)=R-N t$, where $N$ is the Lipschitz constant of $f$ in the interval $[-M, M]$ with $M=\max \left\{\left\|u_{0}\right\|_{\infty},\left\|v_{0}\right\|_{\infty}\right\}$. The Maximum Principle applies:

$$
u_{0} \leq v_{0} \quad \text { implies } \quad u(x, t) \leq v(x, t) \quad \text { in } Q .
$$

Moreover, it is proved that the solution belongs to the class $u \in C\left([0, \infty): L_{\text {loc }}^{1}(\mathbb{R})\right) \cap$ $L^{\infty}(Q)$. We have therefore a semigroup of solutions in $L^{\infty}(\mathbb{R})$, cf. [9].

On the other hand, there is a large literature on the asymptotic behavior of solutions of scalar conservation laws. For instance, in the context of periodic solutions, it is by now well known even for $1 D$ systems of hyperbolic conservation laws, that solutions stabilize around a constant (cf. [7]). In what concerns $L^{1}$-solutions, the asymptotic profiles of solutions are also well known (see for instance [24]). A lot is also known about the stability of shock waves (see for instance [17]).

However, here, we are interested in the possible behavior of all solutions with bounded initial data.

One of the main difficulties to apply the scaling techniques as above is the obtention of compactness. Here we shall use Tartar's results that hold under a suitable non-degeneracy condition on the nonlinearity. The problem of identifying the initial data of the limiting solutions will require also special care.

Theorem 14 Assume that $f$ is not affine on any interval of the real line. Then equation (7.1) generates a regularizing semigroup $S_{t}$ in $L^{\infty}(\mathbb{R})$ satisfying the conditions of Section 4, so that Theorems 9 and 11 apply. The scaling law is now linear, $\sigma(\lambda)=\lambda$.

Proof. Properties (I.i) and (II) are already established. Under the stated conditions on $f$, also called weakly genuine nonlinearity, Tartar [29, 30] proves that bounded sequences of entropy solutions are relatively compact in the strong topology of $L_{l o c}^{p}(Q)$ for any $p<\infty$. Using the local estimate we conclude compactness in the space $C\left([\tau, T]: L_{\text {loc }}^{1}(\mathbb{R})\right)$ for every $0<\tau<T<\infty$. It is immediate to see that the limit $v(x, t)$ of such a sequence is an entropy solution in any time interval $t \geq \tau$ with $\tau>0$.

We still have to examine property (I.ii), i.e., the weak-star continuity of the semigroup map, in the present setting. This is a delicate but essential point. Indeed, the family of rescaled solutions $u_{\lambda}(x, t)$ of Theorem 9 are such that their initial data converge weak-star in $L^{\infty}(\mathbb{R})$ along subsequences,

$$
u_{0, \lambda_{n}} \rightarrow \phi \quad \text { in } \quad L^{\infty}(\mathbb{R}) \text {-ws },
$$


and we want to conclude that $u_{\lambda_{n}}(x, 1)$ converges to $v(x, 1)=S_{1}(\phi)$. We state the general result independently

Lemma 15 Let $\left\{u_{n}\right\}$ be a sequence of bounded entropy solutions of equation (7.1) with initial data $\left\{u_{0, n}\right\}$ which converge to a certain $\phi$ in $L^{\infty}(\mathbb{R})$ weak-star. Then

$$
u_{n} \rightarrow v=S_{t} \phi
$$

in the sense of $C\left([\tau, T]: L_{\text {loc }}^{p}(\mathbb{R})\right)$ for every $0<\tau<T<\infty$ and every $1 \leq p<\infty$.

Proof. (i) By the results of [29] the sequence $u_{n}$ converges in $L_{l o c}^{p}(Q)$ and also a.e. after passing if necessary to a subsequence. The limit $v$ is an entropy solution of the equation. The remaining problem consists of identifying the solution in terms of the limit $\phi$ of the initial data. The classical uniqueness result by Kruzhkov is not enough for our purposes since we do not have strong convergence of $v(t)$ in $L_{\text {loc }}^{1}(\mathbb{R})$ as $t \rightarrow 0$. In other words, we have to discard the possible occurrence of an initial layer of discontinuity. This difficulty has been recently solved by Chen and Rascle [8] who prove uniqueness of entropy solutions of equation (7.1) assuming that the initial data are taken in the sense of measures.

(ii) Here we give an alternative proof which easily follows from the uniqueness result of Liu and Pierre [26].

Proposition 16 Under the assumption that $f: \mathbb{R}_{+} \rightarrow \mathbb{R}_{+}$is Lipschitz continuous and $f(0)=0$ a nonnegative entropy of equation (7.1) such that $u \in L^{\infty}\left((0, T): L^{1}(\mathbb{R})\right) \cap$ $L^{\infty}(\mathbb{R} \times(\tau, T))$, for all $\tau>0$, is uniquely determined by its initial data $u_{0}$ taken in the narrow sense, i.e.,

$$
\lim _{t \rightarrow 0} \int u(x, t) \eta(x) d x=\int u_{0}(x) \eta(x) d x
$$

for every continuous and bounded real function $\eta$.

Indeed, [26] allows the initial data to be finite measures, but this is no concern for us here. Let us note that such a generality forces them to work with nonnegative $f$ and nonnegative solutions, two restrictions that we can dispense with, since we can always displace $u$ into $u+c$ and conserve the equation after a corresponding change in $f$ to satisfy both restrictions.

Let us check the initial data of our limit solution $v$ in the case when it has compact support: passing to the limit in the distributional identity

$$
\int\left(u_{n}(x, t)-u_{0, n}(x)\right) \eta(x) d x=\int_{0}^{t} \int f\left(u_{n}(x, t)\right) \eta(x)_{x} d x d t
$$

valid for every $\eta \in C_{0}^{1}(\mathbb{R})$ we get

$$
\int(v(x, t)-\phi(x)) \eta(x) d x=\int_{0}^{t} \int f(v(x, t)) \eta(x)_{x} d x d t
$$


By density and using the fact that the support of $v$ is uniformly bounded we prove that

$$
\int u(x, t) \eta(x) d x \rightarrow \int u_{0}(x) \eta(x) d x
$$

for every $\eta \in C(\mathbb{R})$. Under these conditions the result of Liu and Pierre [26] and the remark allow us to identify $v$ as the unique entropy solution with initial data $\phi, v(t)=S_{t} \phi$.

(iii) Unfortunately, the condition of belonging to $L^{1}(\mathbb{R})$ is not fulfilled in our case. But the above uniqueness result can still be used, arguing as follows. Let us take a radius $R>0$, a time $T>0$ and work in the cylinder $Z=B(0, R) \times(0, T)$. By the local estimates (7.3) the values of the functions $u_{n}$ on $Z$ are only determined by the initial data in $B\left(0, R_{1}\right)$ with $R_{1}=R+N T$. Therefore, we may replace the data $u_{0, n}$ by functions $u_{0, n}^{\prime}$ which coincide with $u_{0, n}$ on $B\left(0, R_{1}\right)$ and are zero for $|x|>R_{1}$. The solutions $u_{n}^{\prime}(x, t)$ will vanish outside an expanding cone $K=\left\{(x, t):|x|>R_{1}+N t\right\}$ according again to estimate (7.3). Summing up, we may assume that our functions $u_{n}$ belong to the space $C\left([0, \infty): L^{1}(\mathbb{R})\right)$ without altering their value on $Z$.

Remark about Riemann problems. A typical problem in scalar conservation laws is the Riemann Problem, which consists of taking initial data of the step or Heaviside type, (2.2), in which case the solution has the form of a shock or a rarefaction wave, or combinations of these two types. We observe that for data $u_{0}$ whose $G_{\infty}$ limit is a Heaviside function, $G_{\infty}\left(u_{0}\right)=\{H\}$, we obtain convergence of the rescaled solution to the profile at time $t=1$ of the solution of the Riemann problem with data $H(x)$. This is well-known, but we can also consider initial data whose $G_{\infty}$ limit contains any number of Heaviside functions, so that the rescaled orbit oscillates infinitely many times among the corresponding profiles.

7.II Several space dimensions. We look now at the scalar conservation law in several dimensions,

$$
\frac{\partial u}{\partial t}+\sum_{i=1}^{N} \frac{\partial}{\partial x_{i}} f_{i}(u)=0,
$$

where $u(x, t)$ is a scalar function and $f=\left(f_{1}, \cdots, f_{N}\right)$ is a locally Lipschitz continuous vector function. We may assume that $f(0)=0$. The proper concept of solution is given again by the entropy solutions with a formula similar to $(7.2)$, see $[19,20]$. The above theory applies almost literally with the following changes: (i) the compactness property has been established by Lions, Perthame and Tadmor [25] under the following non-degeneracy condition on the non-linearity: for every $(\tau, \zeta) \in \mathbb{R} \times \mathbb{R}^{N}$ which is not $(0,0)$ there holds

$$
\operatorname{meas}\left\{\xi: \tau+\zeta \cdot f^{\prime}(\xi)=0\right\}=0,
$$

where meas refers to the Lebesgue measure in $\mathbb{R}$. Their method relies on the kinetic formulation of the equation, a powerful tool for the several dimensional problem introduced by the authors. 
(ii) The uniqueness of entropy solutions taking bounded data in the weak sense has been recently proved by A. Vasseur [32] using also the kinetic formulation of the equation. The result needs the following regularity on the nonlinearity: $f \in\left(C^{3}(\mathbb{R})\right)^{N}$. We conjecture that this requirement is technical.

Theorem 17 With these two requirements on $f$, Theorem 14 holds with $\mathbb{R}$ replaced by $\mathbb{R}^{N}$ and equation (7.1) by (7.4).

\section{Lack of compactness}

The property of continuity in the weak topology needed for the semigroup is difficult to establish in the case of nonlinear equations unless we also have compactness, i.e., a regularizing semigroup. This last property holds for the PME and the PLE and we imposed conditions of non-degeneracy to have it in the scalar conservation law. However, the Main Asymptotic Formula holds for many equations without compactness. We list below two instances.

I. Linear equations. One of the simplest examples is the case of linear operators, like the scalar conservation law $u_{t}+a u_{x}=0$, even in $N$ dimensions,

$$
u_{t}+\sum_{i=1}^{N} a_{i} u_{x_{i}}=0 .
$$

By means of the explicit representation of solutions it is easy to see that no regularization happens but the semigroup has the desired properties, hence $R_{\infty}=S_{1} G_{\infty}$.

The argument applies of course in the heat equation, but also for the wave equation, where the representation is also explicit and no regularization holds.

II. Scalar Conservation laws. But the result also holds for some nonlinear evolution equations without compactness. Thus, we can study the scalar conservation law (7.1) of previous section with the assumption of local Lipschitz continuity on $f$ but no condition of non-degeneracy. In this case a result of Tartar [30], pag. 202, states that

Weak continuity. Whenever $u_{n}$ is a sequence of entropy solutions of equation (7.1) which are uniformly bounded $\left(\left|u_{n}(x, t)\right| \leq C\right.$ for every $\left.n, x, t\right)$ and

$$
u_{n} \rightarrow u \quad \text { in } L^{\infty}-w^{*} \text {, }
$$

then $f\left(u_{n}\right) \rightarrow f(u)$ in the same sense. On the other hand, $f^{\prime}\left(u_{n}\right)$ converges to $f^{\prime}(u)$ locally in $L^{p}$ strong.

Using this result we can perform the asymptotic study of the scalar conservation law in one dimension for any locally Lipschitz continuous real function $f$. The scaling law is linear, $\sigma(\lambda)=\lambda$. But, as far as we know, the weak-continuity result is not known in several space dimensions, and the asymptotic analysis cannot proceed for equation (7.4) with general Lipschitz $f_{i}$. The question seems difficult and needs further study. 


\section{$9 \quad$ Extending the scope of the theory}

The general setting outlined above for the application to the heat equations and scalar conservation laws in $L^{\infty}\left(\mathbb{R}^{N}\right)$ admits three kinds of extensions where the Main Asymptotic Formula

$$
R_{\infty}\left(u_{0}\right)=S_{1}\left(G_{\infty}\left(u_{0}\right)\right)
$$

still holds when suitably interpreted:

(i) it can be applied to a number of other equations,

(ii) it can be modified to include cases where scale invariance is not strictly respected,

(iii) it can be extended so as to include asymptotic results in other functional settings under the cover of the same formula.

The $L^{1}$ setting. Leaving aside for the moment points (i) and (ii), we will devote this section to show how to adapt the setting of Section 4 to recover the well-known results on asymptotic behaviour of heat equations and conservation laws in the framework of $L^{1}\left(\mathbb{R}^{N}\right)$. Indeed, we can keep the semigroup setting of Sections 3,4 with the following modifications.

- The proper concept of scaling of the data in $L^{1}\left(\mathbb{R}^{N}\right)$ is given by the group $G^{(1)}=\left\{G_{\lambda}^{(1)}\right\}$ acting by

$$
G_{\lambda}^{(1)}(f)=\lambda^{N} f(\lambda x) .
$$

Again, this group is continuous in the weak topology $\sigma\left(L^{1}, L^{\infty}\right)$. We must now note that $L^{1}\left(\mathbb{R}^{N}\right)$ is not closed in this topology, its closure being the space of bounded measures $\mathcal{M}\left(\mathbb{R}^{N}\right)$, which is endowed with the so-called narrow convergence. Indeed, with a definition of $G^{(1)}$-omega limit as in Section 3, it is easy to see that

Lemma 18 For every $f \in L^{\infty}\left(\mathbb{R}^{N}\right)$ we have a simple omega limit,

$$
G_{\infty}^{(1)}(f)=\{c \delta\}
$$

where $c=\int f(x) d x \in \mathbb{R}$ and $\delta$ is the Dirac mass located at $x=0$.

- The equation generates a semigroup acting on $X=L^{1}\left(\mathbb{R}^{N}\right)$. We ask that

(I) The map $S$ is separately continuous in both variables. $X$ is endowed with the weak topology. We also need the semigroup to extend continuously to include the space of measures, or at least, the Dirac deltas.

(II) Scale invariance is formulated in the new setting as

$$
u_{\lambda}(x, t)=\lambda^{N} u\left(\lambda x, \lambda^{\alpha} t\right) .
$$

with suitable exponent $\alpha$. We want $u_{\lambda}$ to be a solution of the equation if $u$ is, i.e., we need the commutation rule

$$
S_{t} G_{\lambda} u_{0}=G_{\lambda} S_{\lambda^{\alpha} t}
$$


Under these conditions we define the rescaled orbit corresponding to a standard orbit $u(\cdot, t)=S_{t} u_{0}$ as

$$
U(y, t)=t^{N / \alpha} u\left(t^{1 / \alpha} y, t\right),
$$

and the renormalized semigroup as $\left(R_{t} u_{0}\right)(y)=U(y, t)$, which implies the identity

$$
R_{t} u_{0}=G_{t^{1 / \alpha}} S_{t} u_{0}
$$

The $R$-omega limit is defined as in Section 4 , and it stays in $L^{1}\left(\mathbb{R}^{N}\right)$ if we make sure that convergence takes place in the strong topology (otherwise, it maight become a measure).

Theorem 19 Under the above assumptions the Main Asymptotic Formula holds true. It means that the $R$-omega limit of the orbits of the semigroup is given by the unique profile at $t=1$ of the fundamental solution of the equation.

If the semigroup is regularizing, i.e., if the map $S_{t}$ sends bounded subsets of $L^{1}\left(\mathbb{R}^{N}\right)$ into relatively compact subsets in the strong topology, then the convergence of the rescaled orbit to the $R_{\infty}$-limit holds in the strong topology.

Applications. Indeed, the four types of equations already considered generate a regularizing semigroup acting on $L^{1}\left(\mathbb{R}^{N}\right)$.

(i) In the case of the heat equation the solution with a Dirac delta exists, is unique, and has the necessary continuity property. It is called the fundamental solution and is given by formula (1.1). Let us remark that the $L^{1}$ norm is conserved by the semigroup, $\int u(x, t) d x=u_{0}(x) d x$. The exponent $\alpha$ in the scaling law is $1 / 2$ as in the $L^{\infty}$ case. The regularizing effect takes $L^{1}\left(\mathbb{R}^{N}\right)$ into bounded subsets of $C^{k}$ for all $k$ (regularity both in space and time). The theorem states the well-known fact that for every $u_{0} \in L^{1}\left(\mathbb{R}^{N}\right)$ we have

$$
\lim _{t \rightarrow \infty} t^{N / 2} u(\sqrt{t} x, t)=c(4 \pi)^{-N / 2} \exp \left(-\frac{x^{2}}{4}\right) .
$$

and the convergence is uniform in $\mathbb{R}^{N}$.

(ii) For the Porous Medium Equation we have a fundamental solution, usually called Barenblatt solution, if $m>m_{*}=(N-1) / N$, and the scaling exponent is

$$
\alpha=N(m-1)+2 .
$$

Again the semigroup is regularizing into $C^{\beta}$ for some $0<\beta<1$ (regularity both in space and time). In this parameter range the theorem applies, cf. [34].

(iii) For the $p$-Laplacian equation we have $\alpha=N(p-2)+p$, and we impose the restriction $p>2 N /(N+1)$ in order to obtain a fundamental solution, also called Barenblatt solution. Regularization occurs into $C^{1, \alpha}$ in space, $C^{\beta}$ in time. 
(iv) For the scalar conservation law we must take $f(u)=u^{p}$ with $p \geq 1$ and then $\alpha=$ $N(p-1)+1$.

In all cases the $L^{1}$ norm is conserved

$$
\int u_{\lambda}(x, t) d x=\int u(x, t) d x=\int u_{0}(x) d x .
$$

Note however that the existence of fundamental entropy solutions for scalar hyperbolic conservation laws is only known in one space dimension. It is unique in the class of constant sign solution, but the occurrence of $N$-waves may produce non-uniqueness phenomena, dependending on whether the nonlinearity $f$ is even or odd (see [26]).

\section{Quasi-invariance}

We may go back to the abstract setting of Section 4 and relax both aspects of the assumption list and still follow the argument advanced in Section 2. We introduce next the concepts of quasi-invariance and limit equation and arrive at a generalized version of the Main Asymptotic Formula.

Quasi-invariance and limit equation. A simple case of this project is exemplified with the viscous approximation to a conservation law, governed by equation

$$
u_{t}+f(u)_{x}=\varepsilon u_{x x},
$$

with $\varepsilon>0$ which approximates and regularizes the solutions of the scalar conservation law (7.1). When we perform the scaling

$$
u_{\lambda}(x, t)=u(\lambda x, \lambda t)
$$

we see that $u_{\lambda}$ does not satisfy the same equation, it satisfies instead

$$
u_{\lambda, t}+f\left(u_{\lambda}\right)_{x}=\frac{\varepsilon}{\lambda} u_{\lambda, x x}
$$

Using the same definition of rescaled orbit and passing to the limit $\lambda \rightarrow \infty$ along a subsequence we get as in Section 7 a limit function $v$ that satisfies the limit equation

$$
v_{t}+f(v)_{x}=0
$$

This is called quasi-invariance, a concept that relates the scaling of equation (10.1) to (7.1). The compactness argument of Tartar applies to the family of equations and we get in the limit an entropy solution with initial data $\phi=\lim _{n} u_{0, \lambda_{n}}$. Summing up, we have

Theorem 20 The main asymptotic formula $R_{\infty}\left(u_{0}\right)=S_{1} G_{\infty}\left(u_{0}\right)$ holds if $R_{\infty}$ is the omega limit of the rescaled orbit and $S_{t}$ is the semigroup generated by the entropy solution of the limit equation (10.2). 
Limit families of equations. The situation can be more involved when the limit equation is not unique. Such a situation arises in Homogenization Problems. We consider next the heat equation

$$
\rho(x) u_{t}=\Delta u,
$$

where $\rho \in L^{\infty}\left(\mathbb{R}^{N}\right)$ is such that $0<c_{1} \leq \rho(x) \leq c_{2}$. Let $S_{t}\left(u_{0} ; \rho\right)$ denote the heat semigroup with weight $\rho$. When we re-scale the solutions by means of formula (2.3)

$$
u_{\lambda}(x, t)=u\left(\lambda x, \lambda^{2} t\right)
$$

we get the equation satisfied by $u_{\lambda}$

$$
\rho_{\lambda}(x) u_{\lambda, t}=\Delta u_{\lambda}
$$

where $\rho_{\lambda}(x)=\rho(\lambda x)$. Let us now pass to a subsequence where

$$
u_{0}(\lambda x) \rightarrow \phi, \quad \rho_{\lambda} \rightarrow a, \quad u_{\lambda}(x, t) \rightarrow v(x, t) .
$$

We easily see that such a limit $v$ satisfies the homogenized equation

$$
a(x) v_{t}=\Delta v(x),
$$

where $a$ ranges over all the elements in $G_{\infty}(\rho)$. In this case we have a situation where, instead of a limit equation, there exists in general a family of limit equations. Note however that in the important case of periodic coefficients the limit equation is unique since $a$ is a constant, the average of $\rho$. This happens also for almost periodic coefficients.

We have then the following theorem.

Theorem 21 The set of accumulation points of the rescaled orbit satisfies

$$
R_{\infty}\left(u_{0}\right) \subset\left\{S_{1}(\phi ; a): \phi \in G_{\infty}\left(u_{0}\right), a \in G_{\infty}(\rho)\right\} .
$$

Equality of the sets in the theorem is not necessarily true since $\rho$ and $u_{0}$ may be coupled. If we take for instance $u_{0}=\rho$ then we only obtain $R$-omega limits of the form $S_{1}(a ; a)$. Depending on how independent are the oscillations of $u_{0}$ and $\rho$ we may obtain larger $R$-omega limit sets, even equality in the formula.

About an asymptotic theory with quasi-invariance. The general evolution setting introduced in Section 4 for invariant semigroups can be easily modified to cover the case of quasi-invariant semi-groups and their limits according to the above two examples. Since there are no special novelties we leave the details to the reader and delay the detailed study of this aspect until other relevant examples have been examined. Actually, the ideas of quasi-invariance can be applied to study the asymptotic behavior of numerous processes appearing in the physical sciences. This is the case for equations combining nonlinear diffusion, reaction and convection. 
Diffusion-convection equations of the form

$$
u_{t}=\Delta u+\frac{\partial}{\partial x_{1}} f(u)
$$

with $f$ is a scalar function, are studied in [13], [14] in the setting of $L^{1}$ data. Convergence of the rescaled solutions is proved towards the solutions of the equation with partial diffusivity

$$
u_{t}=\Delta^{\prime} u+\frac{\partial}{\partial x_{1}} f(u)
$$

where $\Delta^{\prime}$ denotes the Laplacian with respect to the variables $x^{\prime}=\left(x_{2}, \cdots, x_{n}\right)$. In order to extend the results of this paper to this model for $L^{\infty}\left(\mathbb{R}^{N}\right)$ data we need a different scaling

$$
u_{\lambda}(x, t)=u\left(\lambda x_{1}, \sqrt{\lambda} x^{\prime}, \lambda t\right) .
$$

Proving the asymptotic result should offer only difficulties related to compactness and identification of the limit solution (cf. Section 7). Besides, the scaling of the initial data in Section 3 has to be adapted to this anisotropic formula. In any case, Theorem 2 still holds in this setting, which guarantees the occurrence of the complexity we want to describe.

Further interesting lines of application concern the equations of fluid mechanics and the kinetic equations, but the technical difficulties are greater.

\section{Acknowledgments}

The authors are grateful to P. L. Lions, M. Rascle, D. Serre and L. Tartar for useful information about conservation laws. The work of the first author was supported by DGES Grant PB94-0153 and EU Programme TMR FMRX-CT98-0201, and the second author by DGES Grant PB96-0663 and TMR Programme Homogenization and Multiple Scales. The first author also acknowledges partial support from TICAM, Univ. of Texas at Austin.

\section{References}

[1] D. Aronson, Ph. BÉnilan, A nonlinear heat equation with singular diffusivity, Commun. in partial differ. equations, 13 (1979), 985-1039.

[2] Ph. BÉnilan, "Equations d'évolution dans un espace de Banach quelconque et applications", Thesis, Orsay, 1972.

[3] Ph. BÉnilan, S.N. Kruzhkov, Conservation laws with continuous flux functions, Nonlinear Diff. Eqns. Applic. (NODEA), 3 (4) (1996), 395-419. 
[4] H. Bohr, "Almost periodic functions", Chelsea, 1947.

[5] H. Brezis, Analyse Fonctionnelle, Masson, Paris, 1983.

[6] E. Chasseigne, J.L. Vazquez, Extended Solutions for Fast Diffusion Equations in Optimal Classes of Data I. The Cauchy Problem, Preprint (2000).

[7] G.Q. Chen, H. Frid, Initial layers and uniqueness of weak entropy solutions to hyperbolic conservation laws, Arch. Rat. Mech. Anal. 153 (2000 ), 205-220.

[8] G.Q. Chen, M. RAscle, Initial layers and uniqueness of weak entropy solutions to hyperbolic laws, Arch. Rat. Mech. Anal., 153 (2000), 205-220.

[9] M.G. CRANDAlL, The semigroup approach to first-order quasilinear equations in several space variables, Israel J. Math. 12 (1972), 108-132.

[10] E. DiBenedetto, "Degenerate Parabolic Equations", Series Universitext, Springer-Verlag, New York, 1993.

[11] E. Di Benedetto, A. Friedman, Hölder estimates for non-linear degenerate parabolic systems, J. Reine Angew. Math., 357 (1985), 1-22. Addendum, J. Reine Angew. Math., 363 (1985), 217-220.

[12] J. Duonndikomtxea, E. Zuazua, Moments, masses de Dirac et décomposition de fonctions, C. R. Acad. Sci. Paris., 315 (1992), 693-698.

[13] M. Escobedo, J.L. Vazquez, E. ZuazuA, A diffusion-convection equation in several space variables, Indiana Univ. Math. J., 42 (1993), 1413-1440.

[14] M. Escobedo, J. L. Vazquez, E. Zuazua, Entropy solutions for diffusionconvection equations with partial diffusivity, Transactions Amer. Math. Soc., 343 (1994), 829-842.

[15] M. Escobedo, E. ZuAzuA, Long time behavior for convection-diffusion equations, SIAM J. Math. Anal., 28 (1997), 570-594.

[16] J. R. Esteban, J. L. VazQuez, Régularité des solutions positives de l'équation parabolique p-laplacienne, Comptes Rendus Acad. Sciences Paris I, 310 (1990), 105-110.

[17] H. Freistuhler AND D. SERRE, $L^{1}$-stability of shock waves in scalar viscous conservation laws, Comm. Pure Appl. Math., 51 (1998), 291-301.

[18] E. Godlewski, P.A. Raviart, "Hyperbolic Systems of Conservation Laws", Mathématiques \& Appl. 3-4, Ellipses, Paris, 1991. 
[19] S. N. KRuzhKov, Generalized solutions of the Cauchy problem in the large for nonlinear equations of first order, Dokl. Akad. Nauk SSSR 187, 1 (1969), 29-32 (english transl. in Soviet Math. Dokl. 10, 1969)

[20] S. N. KRuzhKov, First-order quasilinear equations in several independent variables, Mat. Sbornik 81, 2 (1970), 228-255 (english transl. Math. USSR Sb. 10 (1970), 217-243).

[21] J. K. Hale, "Asymptotic behavior of dissipative systems", Math. Surveys and Monographs 25, Amer. Math. Soc., Providence, 1988.

[22] O.A. Ladyzhensakaya, A dynamical system generated by the Navier-Stokes equation, Zap. Nauchn. Sem. Leningrad Otdel. Mat. Inst. Steklov, 27 (1972), 91115 .

[23] P. D. LAx, Hyperbolic systems of conservation laws II, Comm. Pure Appl. Math., 10 (1957), 537-566.

[24] T.P. LiU, "Hyperbolic and viscous conservation laws", CBMSS-NSF Regional Conference Series in Applied Mathematics 72, SIAM, Philadelphia, 1999.

[25] P.L. Lions, B. Perthame, E. TAdmor, A kinetic formulation of multidimensional scalar conservation laws and related equations, J. Am. Math. Soc., 7 (1994), 169-191.

[26] T.P. Liu, M. PierRe, Source solutions and asymptotic behaviour in conservation laws, J. Diff. Eqns., 51 (1984), 419-441.

[27] J. Orteg A, E. ZuAzUA, Large time behavior in $\mathbb{R}^{N}$ for linear parabolic equations with periodic coefficients, Asymptotic Analysis, 22 (2000), 51-85.

[28] D. Serre, "Systèmes de lois de conservation", II, Diderot Ed., Paris, 1996.

[29] L. TARTAR, Une nouvelle méthode de résolution d'équations aux dérivées partielles non linéaires, Lecture Notes in Math. 665, Springer, Berlin, 1977, pp. 228-241.

[30] L. TArTar, Compensated compactness and applications to Partial Differential Equations, in "Nonlinear Analysis and Mechanics: Heriot-Watt Symposium", vol. IV, Pitman, San Francisco, 1979. pp. 136-212.

[31] R. TÉmam, "Infinite dimensional dynamical systems in mechanics and physics", Springer Verlag, 1988.

[32] A. VASSEuR, Strong traces for solutions to the multi-dimensional conservation laws, preprint Univ. Nice, 2000. 
[33] J.L. VAzQUEz, Nonexistence of solutions for nonlinear heat equations of fastdiffusion type, Journal Math. Pures et Appliquées, 71 (1992), 503-526.

[34] J.L. VAzQuez, "Asymptotic behaviour for the Porous Medium Equation in the whole space" Ph. D. Course, UAM 1996/97. See UAM address http:// www.adi.uam.es/ ${ }^{\text {jvazquez }}$

EMAIL ADDRESSES:

juanluis.vazquez@uam.es

zuazua@eucmax.sim.ucm.es 